J. DIFFERENTIAL GEOMETRY

58 (2001) 501-534

\title{
COMPLETE MANIFOLDS WITH POSITIVE SPECTRUM
}

\author{
PETER LI \& JIAPING WANG
}

\begin{abstract}
In this paper, we studied complete manifolds whose spectrum of the Laplacian has a positive lower bound. In particular, if the Ricci curvature is bounded from below by some negative multiple of the lower bound of the spectrum, then we established a splitting type theorem. Moreover, if this assumption on the Ricci curvature is only valid outside a compact subset, then the manifold must have only finitely many ends with infinite volume. Similar type theorems are also obtained for complete Kähler manifolds.
\end{abstract}

\section{Introduction}

In a recent work of Witten-Yau [17], they proved that if $M^{n}$ is a conformally compact, Einstein, $n \geq 3$ dimensional manifold whose boundary has positive Yamabe constant, then the homology group $H_{n-1}(M, Z)=0$. In particular, $M$ must have only one end. Let us recall that a manifold $M$ is conformally compact if its complete metric is of the form

$$
d s^{2}=\rho^{-2} d s_{0}^{2}
$$

where $d s_{0}^{2}$ is some background metric defined on the manifold with boundary $\bar{M}=M \cup \partial M$ and $\rho$ is a defining function satisfying

$$
\rho=0 \quad \text { on } \partial M
$$

and

$$
d \rho \neq 0 \quad \text { on } \partial M \text {. }
$$

The first author was partially supported by NSF grant \#DMS-9971418 and an Earmarked grant of Hong Kong. The second author was partially supported by NSF grant \#DMS-0072181.

Received August 5, 2001. 
A direct computation implies that such a manifold must have sectional curvature asymptotically given by $-|d \rho|^{2}$ at infinity. In addition, if the manifold is assumed to be Einstein then $M$ must have asymptotically constant negative curvature. The Witten-Yau theorem resolved an outstanding issue in the AdS/CFT correspondence and effectively ruled out the existence of worm holes. In a preprint of Cai-Galloway [3], they relaxed the assumption that $\partial M$ has positive Yamabe constant to allow nonnegative Yamabe constant.

In his thesis, [15] and [16], X. Wang generalized the above theorem of Witten-Yau and Cai-Galloway and proved the following theorem.

Theorem 0.1 (X. Wang). Let $M^{n}$ be an $n$-dimensional $(n \geq 3)$, conformally compact manifold with Ricci curvature bounded from below by

$$
\operatorname{Ric}_{M} \geq-(n-1) \text {. }
$$

Let $\lambda_{1}(M)$ denote the lower bound of the spectrum of the Laplacian on M. If

$$
\lambda_{1}(M) \geq n-2
$$

then either

(1) $H^{1}\left(L^{2}(M)\right)=0$; or

(2) $M=\mathbb{R} \times N$ with the warped product metric $d s^{2}=d t^{2}+\cosh ^{2} t d s_{N}^{2}$, where $N$ is a compact manifold with Ricci curvature bounded from below by

$$
\operatorname{Ric}_{N} \geq-(n-2) \text {. }
$$

In particular, $M$ either has only one end or it must be a warped product given as above.

The conclusion on the number of ends follows from Mazzeo's theorem [14] which identifies the $L^{2}$-cohomology group $H^{1}\left(L^{2}(M)\right)$ with the relative cohomology group $H^{1}(M, \partial M)$ for conformally compact manifolds. Moreover, according to a theorem of Lee [5], which asserts that if a conformally compact, Einstein manifold $M^{n}$ has nonnegative Yamabe constant for its boundary, then $\lambda_{1}(M)=\frac{(n-1)^{2}}{4}$, the aforementioned theorem of Wang indeed implies the theorems of Witten-Yau and CaiGalloway. Obviously, the warped product manifold does not have nonnegative Yamabe constant for its boundaries. 
Note that the pair of conditions (0.1) and (0.2) can be rewritten as

$$
\operatorname{Ric}_{M} \geq-\frac{(n-1) \lambda_{1}(M)}{n-2}
$$

and

$$
\lambda_{1}(M)>0 .
$$

When written in this form then one can identify the class of manifolds with nonnegative Ricci curvature as those satisfying $(0.3)$ and $\lambda_{1}(M)=$ 0 . Indeed these two classes of manifolds are identical because manifolds with nonnegative Ricci curvature must have $\lambda_{1}(M)=0$.

The main purpose of this article is to study the class of complete manifolds satisfying conditions (0.3) and (0.4). In particular, we will generalize Wang's theorem to manifolds which are not necessarily conformally compact. In Theorem 2.1, we showed that if a manifold of dimension $n \geq 3$ satisfies the conditions (0.3) and (0.4), then either it must have only one infinite volume end or it is a warped product $\mathbb{R} \times N$ as described in Theorem 0.1 .

Observe that if $M$ is conformally compact, then all its ends must have infinite volume, hence Wang's theorem follows as a corollary. We would also like to point out that we cannot prove the vanishing of $H^{1}\left(L^{2}(M)\right)$. Moreover, since $M$ might not be conformally compact, we do not have Mazzeo's theorem identifying $H^{1}\left(L^{2}(M)\right) \simeq H^{1}(M, \partial M)$. However, we will use a theorem of Tam and the first author [12] (also see [10]), which allows us to estimate the number of ends using harmonic functions.

In Theorem 3.1, we also prove that if the curvature assumption (0.3) is satisfied only at infinity with a strict inequality, then the manifold must have finitely many ends with infinite volume. Moreover, the number of ends can be estimated in terms of the geometry of $M$.

At this point, we like to point out the advantage of viewing Theorem 2.1 using conditions (0.3) and (0.4) instead of (0.1) and (0.2). As mentioned earlier, since the class of manifolds with nonnegative Ricci curvature can be identified as those manifolds satisfying (0.3) and $\lambda_{1}(M)=0$, Theorem 2.1 can be viewed as a parallel theory to the splitting theorem of Cheeger-Gromoll [1]. Also, a rephrased version of Cheng's theorem [2] asserts that the class of complete noncompact manifolds satisfying (0.4) and

$$
\operatorname{Ric}_{M} \geq-\frac{4 \lambda_{1}(M)}{n-1}+\epsilon
$$


for some $\epsilon>0$, is empty. Moreover, the equality condition

$$
\operatorname{Ric}_{M}=-\frac{4 \lambda_{1}(M)}{n-1}
$$

is satisfied by (but not only) the standard hyperbolic space $\mathbb{H}^{n}$. Theorem 2.1 primarily addresses those cases when the lower bound of the Ricci curvature is in the range $\left[-\frac{(n-1) \lambda_{1}(M)}{n-2},-\frac{4 \lambda_{1}(M)}{n-1}\right]$. Theorem 3.1 can also be viewed as a parallel development to the finitely many ends theorem of Tam and the first author [12] for manifolds with Ricci curvature satisfying

$$
\operatorname{Ric}_{M}(x) \geq-C r^{-2}(x) .
$$

In $\S 4$, we prove a vanishing theorem (Theorem 4.2) and a finiteness theorem (Theorem 4.3) for $H^{1}\left(L^{2}(M)\right)$. In these cases, the assumption on the bound of the Ricci curvature is stronger than that of Theorem 2.1 and Theorem 3.1. However, the conclusion is also stronger because the vanishing and finiteness of $H^{1}\left(L^{2}(M)\right)$ will imply the uniqueness and finiteness of infinite volume ends, respectively.

When the manifold $M$ is a complete Kähler manifold with complex dimension $m$, then one can improve Theorem 2.1 and Theorem 3.1 by relaxing the Ricci curvature assumption (see Theorem 5.1 and Theorem 5.2). In this case, the warped product does not exist due to the Kählerian condition. Although our argument is probably not sharp in this setting, however we suspect that there is a complex version of our splitting theorem analogous to Theorem 2.1.

It is also worth pointing out that in our preparation $(\S 1)$ for proving the aforementioned theorems, we developed sharp $L^{2}$-estimates for a class of harmonic functions constructed in [12] for complete manifolds with positive spectrum. These estimates are valid without any additional assumption, such as curvature bounds, on the manifold. By using these estimates, we will prove in Theorem 1.4 that a complete manifold with $\lambda_{1}=1$ must have volume growth bounded from below by

$$
V(R) \geq C \exp (2 R)
$$

Moreover, this estimate can be localized to an end. In this case if an end $E$ of a complete manifold has $\lambda_{1}=1$, then either it has volume growth bounded from below by

$$
V_{E}(R) \geq C \exp (2 R)
$$


or it must be of finite volume with volume decay bounded from above by

$$
V_{E}(\infty)-V_{E}(R) \leq C \exp (-2 R)
$$

Note that both the growth and decay estimates are sharp. The growth estimate is realized by the standard hyperbolic space $\mathbb{H}^{n}$, while the decay estimate is realized by the hyperbolic cusp. Moreover, Example 2.2 in dimension 3 also realizes the sharp estimates.

The first author would like to thank Tom Wan for pointing out Wang's theorem to him. In a recent preprint [6] of Leung and Wan, they have generalized Wang's vanishing theorem for $L^{2}$ harmonic 1forms on a conformally compact manifold to harmonic maps into nonpositively curved manifolds with finite total energy. The authors would also like to thank the referee for suggesting a simplification of the proof of Lemma 1.1.

\section{Preliminary estimates}

Let us first recall (see [12] and [10]) that an end $E$ of a complete manifold $M$ is non-parabolic means that $E$ admits a positive Green's function with Neumann boundary condition. In the previous work of the first author and Tam in [12], they proved that the number of nonparabolic ends of a complete manifold is bounded from above by the dimension of the space of bounded harmonic functions with finite Dirichlet integral. On the other hand, in the work of Cao-Shen-Zhu [4] (see Corollary 4 in [13]), they observed that if an end of a manifold has a positive lower bound for the spectrum of the Laplacian, then the end must either be non-parabolic or has finite volume. These two facts together allow us to estimate the number of ends with infinite volume on a manifold with positive spectrum by estimating the dimension of the space of bounded harmonic functions with finite Dirichlet integral. Unfortunately, we do not yet know how to estimate the dimension of this space. However, we managed to estimate those harmonic functions which were constructed in the proof of the Li-Tam theorem [12]. This is sufficient to estimate the number of infinite volume ends.

Let us give an outline of the proof for the theorem of Li-Tam. For our purpose, let us assume that $M$ has at least 2 non-parabolic ends, otherwise there is nothing to prove. Suppose $R_{0}>0$ is sufficiently large so that $M \backslash B_{p}\left(R_{0}\right)$ has at least 2 disjoint non-parabolic ends $E_{1}$ and 
$E_{2}$. For the sake of convenience, if $E$ is an end of $M$ then let us denote

$$
E(R)=E \cap B_{p}(R)
$$

and

$$
\partial E(R)=E \cap \partial B_{p}(R) .
$$

We will construct a nonconstant bounded harmonic function with finite Dirichlet integral adopted for the end $E_{1}$. For $R \geq R_{0}$ we will solve the following Laplace equation with the given boundary value. Let $f_{R}$ be the solution of

$$
\begin{aligned}
\Delta f_{R}=0 & \text { on } B_{p}(R), \\
f_{R}=1 & \text { on } \partial E_{1}(R),
\end{aligned}
$$

and

$$
f_{R}=0 \quad \text { on } \partial B_{p}(R) \backslash E_{1} .
$$

Since $R \geq R_{0}$, clearly $\partial E_{2}(R) \subset\left(\partial B_{p}(R) \backslash E_{1}\right)$. Due to the assumption that both $E_{1}$ and $E_{2}$ are non-parabolic, the sequence of functions $\left\{f_{R}\right\}$ must have a subsequence that converges to a harmonic function $f$ defined on $M$ which has the property that $\sup _{M} f=\sup _{E_{1}} f=1$ and $\inf _{M} f=\inf _{E_{i}} f=0$ for any non-parabolic ends $E_{i}$ with $i \neq 1$. In particular, $f$ is bounded and also it has finite Dirichlet integral. Obviously, we can use this construction on each non-parabolic end and obtain as many linearly independent harmonic functions, including the constant function, as the number of non-parabolic ends. Let us denote the space spanned by those harmonic functions by $K$. By estimating the dimension of $K$, we will be able to estimate the number of non-parabolic ends. If $M$, or all its ends, have positive $\lambda_{1}$, then

$$
\operatorname{dim} K \geq \text { number of infinite volume ends. }
$$

In the following lemma, we will first obtain a decay estimate for the functions in $K$. Throughout the rest of the paper, we will denote the volume of the set $E(R)$ by $V_{E}(R)$ and the area of $\partial E(R)$ by $A_{E}(R)$. Recall that $\lambda_{1}(E)$, the bottom of the $L^{2}$ spectrum of the Laplacian on $E$ satisfying Dirichlet boundary conditions on $\partial E$, may be characterized alternatively as

$$
\lambda_{1}(E) \int_{E} \phi^{2} \leq \int_{E}|\nabla \phi|^{2}
$$

for all compactly supported smooth function $\phi$ on $E$. 
Lemma 1.1. Let $M$ be a complete Riemannian manifold. Suppose $E$ is an end of $M$ such that $\lambda_{1}(E)>0$. Then for any harmonic function $f \in K$, there exists a constant a such that $f-$ a must be in $L^{2}(E)$. Moreover, the function $f-a$ must satisfy the decay estimate

$$
\int_{E(R+1) \backslash E(R)}(f-a)^{2} \leq C \exp \left(-2 \sqrt{\lambda_{1}(E)} R\right)
$$

for some constant $C>0$ depending on $f, \lambda_{1}(E)$ and $n$.

Proof. It suffices to prove the lemma for those $f$ constructed above because the decay property is preserved under linear combinations. By scaling the metric, we may assume that $\lambda_{1}(E)=1$. Let $f_{R}$ be a sequence of harmonic functions constructed above that converges to $f$. For a fixed end $E$, since $f_{R}$ has boundary value either 0 or 1 on $\partial E(R)$, we will consider either the function $f_{R}$ or $1-f_{R}$. For simplicity, let us assume that $f_{R}$ has boundary value 0 on $\partial E(R)$. We will then show that the lemma holds with $a=0$ and

$$
\int_{E(R+1) \backslash E(R)} f^{2} \leq C \exp (-2 R) .
$$

To do so, we first verify that for any $0<\delta<1$,

$$
\int_{E} \exp (2 \delta r) f^{2} \leq \frac{C}{(1-\delta)^{2}}
$$

In particular, function $f$ is in $L^{2}(E)$. Indeed, let $\phi$ be a nonnegative cut-off function defined by

$$
\phi(x)=\frac{r(x)-R_{0}}{R_{0}} \quad \text { on } E\left(2 R_{0}\right) \backslash E\left(R_{0}\right),
$$

and

$$
\phi=1 \quad \text { on } E \backslash E\left(2 R_{0}\right),
$$

where $r(x)$ is the geodesic distance to the fixed point $p$. Then integration by parts yields 


$$
\begin{aligned}
& \int_{E(R)}\left|\nabla\left(\phi \exp (\delta r) f_{R}\right)\right|^{2} \\
& =\int_{E(R)}|\nabla(\phi \exp (\delta r))|^{2} f_{R}^{2} \\
& +2 \int_{E(R)} \phi \exp (\delta r) f_{R}\left\langle\nabla(\phi \exp (\delta r)), \nabla f_{R}\right\rangle \\
& +\int_{E(R)}(\phi \exp (\delta r))^{2}\left|\nabla f_{R}\right|^{2} \\
& =\int_{E(R)}|\nabla(\phi \exp (\delta r))|^{2} f_{R}^{2}+\frac{1}{2} \int_{E(R)}\left\langle\nabla\left(\phi^{2} \exp (2 \delta r)\right), \nabla\left(f_{R}^{2}\right)\right\rangle \\
& +\int_{E(R)} \phi^{2} \exp (2 \delta r)\left|\nabla f_{R}\right|^{2} \\
& =\int_{E(R)}|\nabla(\phi \exp (\delta r))|^{2} f_{R}^{2}-\frac{1}{2} \int_{E(R)} \phi^{2} \exp (2 \delta r) \Delta\left(f_{R}^{2}\right) \\
& +\int_{E(R)} \phi^{2} \exp (2 \delta r)\left|\nabla f_{R}\right|^{2} \\
& =\int_{E(R)}|\nabla(\phi \exp (\delta r))|^{2} f_{R}^{2} \\
& \leq(1+\epsilon) \int_{E(R)} \phi^{2}|\nabla \exp (\delta r)|^{2} f_{R}^{2} \\
& +\left(1+\frac{1}{\epsilon}\right) \int_{E(R)} \exp (2 \delta r)|\nabla \phi|^{2} f_{R}^{2} \\
& \leq(1+\epsilon) \delta^{2} \int_{E(R)} \phi^{2} \exp (2 \delta r) f_{R}^{2} \\
& +\left(1+\frac{1}{\epsilon}\right) \frac{1}{R_{0}^{2}} \int_{E\left(2 R_{0}\right) \backslash E\left(R_{0}\right)} \exp (2 \delta r) f_{R}^{2} .
\end{aligned}
$$

Combining with the assumption that $\lambda_{1}(E)=1$, we have

$$
\int_{E(R)} \phi^{2} \exp (2 \delta r) f_{R}^{2} \leq \int_{E(R)}\left|\nabla\left(\phi \exp (\delta r) f_{R}\right)\right|^{2},
$$

hence

$$
\begin{aligned}
& \left(1-(1+\epsilon) \delta^{2}\right) \int_{E(R)} \phi^{2} \exp (2 \delta r) f_{R}^{2} \\
& \leq\left(1+\frac{1}{\epsilon}\right) \frac{1}{R_{0}^{2}} \int_{E\left(2 R_{0}\right) \backslash E\left(R_{0}\right)} \exp (2 \delta r) f_{R}^{2} .
\end{aligned}
$$


For $0<\delta<1$, we can choose $\epsilon=\frac{1-\delta}{\delta}$ and obtain the estimate

$$
(1-\delta)^{2} \int_{E(R) \backslash E\left(2 R_{0}\right)} \exp (2 \delta r) f_{R}^{2} \leq \frac{1}{R_{0}^{2}} \int_{E\left(2 R_{0}\right) \backslash E\left(R_{0}\right)} \exp (2 \delta r) f_{R}^{2} .
$$

Letting $R \rightarrow \infty$, this implies

$$
(1-\delta)^{2} \int_{E \backslash E\left(2 R_{0}\right)} \exp (2 \delta r) f^{2} \leq \frac{1}{R_{0}^{2}} \int_{E\left(2 R_{0}\right) \backslash E\left(R_{0}\right)} \exp (2 \delta r) f^{2},
$$

which can be written as

$$
\int_{E} \exp (2 \delta r) f^{2} \leq \frac{C}{(1-\delta)^{2}}
$$

Our next step is to improve this estimate by trying to set $\delta=1$ in the preceding argument. Note that for any function $\psi$ with compact support in $E$, since $\lambda_{1}(E)=1$,

$$
\begin{aligned}
\int_{E} \psi^{2} \exp (2 r) f^{2} \leq & \int_{E} \mid \nabla\left(\left.\psi \exp (r) f\right|^{2}\right. \\
= & \int_{E}|\nabla \psi|^{2} \exp (2 r) f^{2}+2 \int_{E} \psi \exp (2 r)\langle\nabla \psi, \nabla r\rangle f^{2} \\
& +\int_{E} \psi^{2} \exp (2 r) f^{2},
\end{aligned}
$$

hence we have

$$
-2 \int_{E} \psi \exp (2 r)\langle\nabla \psi, \nabla r\rangle f^{2} \leq \int_{E}|\nabla \psi|^{2} \exp (2 r) f^{2} .
$$

For $R_{0}<R_{1}<R$, let us choose $\psi$ to be

$$
\psi(x)= \begin{cases}\frac{r(x)-R_{0}}{R_{1}-R_{0}} & \text { on } E\left(R_{1}\right) \backslash E\left(R_{0}\right) \\ \frac{R-r(x)}{R-R_{1}} & \text { on } E(R) \backslash E\left(R_{1}\right) .\end{cases}
$$

We conclude that

$$
\begin{aligned}
\frac{2}{R-R_{1}} & \int_{E(R) \backslash E\left(R_{1}\right)}\left(\frac{R-r(x)}{R-R_{1}}\right) \exp (2 r) f^{2} \\
\leq & \frac{1}{\left(R_{1}-R_{0}\right)^{2}} \int_{E\left(R_{1}\right) \backslash E\left(R_{0}\right)} \exp (2 r) f^{2} \\
& +\frac{1}{\left(R-R_{1}\right)^{2}} \int_{E(R) \backslash E\left(R_{1}\right)} \exp (2 r) f^{2} \\
& +\frac{2}{\left(R_{1}-R_{0}\right)^{2}} \int_{E\left(R_{1}\right) \backslash E\left(R_{0}\right)}\left(r-R_{0}\right) \exp (2 r) f^{2}
\end{aligned}
$$


On the other hand, for any fixed $0<t<R-R_{1}$, since

$$
\begin{aligned}
\frac{2 t}{\left(R-R_{1}\right)^{2}} & \int_{E(R-t) \backslash E\left(R_{1}\right)} \exp (2 r) f^{2} \\
& \leq \frac{2}{\left(R-R_{1}\right)^{2}} \int_{E(R) \backslash E\left(R_{1}\right)}(R-r(x)) \exp (2 r) f^{2},
\end{aligned}
$$

we deduce that

$$
\begin{aligned}
\frac{2 t}{\left(R-R_{1}\right)^{2}} & \int_{E(R-t) \backslash E\left(R_{1}\right)} \exp (2 r) f^{2} \\
\leq & \left(\frac{2}{R_{1}-R_{0}}+\frac{1}{\left(R_{1}-R_{0}\right)^{2}}\right) \int_{E\left(R_{1}\right) \backslash E\left(R_{0}\right)} \exp (2 r) f^{2} \\
& +\frac{1}{\left(R-R_{1}\right)^{2}} \int_{E(R) \backslash E\left(R_{1}\right)} \exp (2 r) f^{2} .
\end{aligned}
$$

Observe that if we take $R_{1}=R_{0}+1, t=1$ and set

$$
g(R)=\int_{E(R) \backslash E\left(R_{0}+1\right)} \exp (2 r) f^{2},
$$

then the inequality (1.2) can be written as

$$
g(R-1) \leq C R^{2}+\frac{1}{2} g(R)
$$

where

$$
C=3 \int_{E\left(R_{0}+1\right) \backslash E\left(R_{0}\right)} \exp (2 r) f^{2}
$$

is independent of $R$. Iterating this inequality, we obtain for any positive integer $k$ and $R \geq 1$

$$
\begin{aligned}
g(R) & \leq C \sum_{i=1}^{k} \frac{(R+i)^{2}}{2^{i-1}}+2^{-k} g(R+k) \\
& \leq C R^{2} \sum_{i=1}^{\infty} \frac{(1+i)^{2}}{2^{i-1}}+2^{-k} g(R+k) \\
& \leq C R^{2}+2^{-k} g(R+k) .
\end{aligned}
$$

However, our previous estimate (1.1) asserts that

$$
\int_{E} \exp (2 \delta r) f^{2} \leq \frac{C}{(1-\delta)^{2}}
$$


for any $\delta<1$. This implies that

$$
\begin{aligned}
g(R+k) & =\int_{E(R+k) \backslash E\left(R_{0}+1\right)} \exp (2 r) f^{2} \\
& \leq \exp (2(R+k)(1-\delta)) \int_{E(R+k) \backslash E\left(R_{0}+1\right)} \exp (2 \delta r) f^{2} \\
& \leq C(1-\delta)^{-2} \exp (2(R+k)(1-\delta)) .
\end{aligned}
$$

Hence,

$$
2^{-k} g(R+k) \rightarrow 0
$$

as $k \rightarrow \infty$ by choosing $2(1-\delta)<\ln 2$. This proves the estimate that

$$
g(R) \leq C R^{2} \text {. }
$$

By adjusting the constant, we have

$$
\int_{E(R)} \exp (2 r) f^{2} \leq C R^{2}
$$

for all $R \geq R_{0}$.

Using inequality (1.2) again and by choosing $R_{1}=R_{0}+1$ and $t=\frac{R}{2}$ this time, we conclude that

$$
R \int_{E\left(\frac{R}{2}\right) \backslash E\left(R_{0}+1\right)} \exp (2 r) f^{2} \leq C R^{2}+\int_{E(R) \backslash E\left(R_{0}+1\right)} \exp (2 r) f^{2} .
$$

However, applying the estimate (1.3) to the second term on the right hand side, we have

$$
\int_{E\left(\frac{R}{2}\right) \backslash E\left(R_{0}+1\right)} \exp (2 r) f^{2} \leq C R .
$$

Therefore, for $R \geq R_{0}$,

$$
\int_{E(R)} \exp (2 r) f^{2} \leq C R
$$

We are now ready to prove the lemma by using (1.4). Setting $t=2$ and $R_{1}=R-4$ in (1.2), we obtain

$$
\begin{aligned}
& \int_{E(R-2) \backslash E(R-4)} \exp (2 r) f^{2} \\
& \quad \leq\left(\frac{8}{R-R_{0}-4}+\frac{4}{\left(R-R_{0}-4\right)^{2}}\right) \int_{E(R-4) \backslash E\left(R_{0}\right)} \exp (2 r) f^{2} \\
& \quad+\frac{1}{4} \int_{E(R) \backslash E(R-4)} \exp (2 r) f^{2} .
\end{aligned}
$$


According to (1.4), the first term of the right hand side is bounded by a constant. Hence, the above inequality can be rewritten as

$$
\int_{E(R-2) \backslash E(R-4)} \exp (2 r) f^{2} \leq C+\frac{1}{3} \int_{E(R) \backslash E(R-2)} \exp (2 r) f^{2} .
$$

Iterating this inequality $k$ times, we arrive at

$$
\begin{aligned}
& \int_{E(R+2) \backslash E(R)} \exp (2 r) f^{2} \\
& \leq C \sum_{i=0}^{k-1} 3^{-i}+3^{-k} \int_{E(R+2(k+1)) \backslash E(R+2 k)} \exp (2 r) f^{2} .
\end{aligned}
$$

However, using (1.4) again, we conclude that the second term is bounded by

$$
3^{-k} \int_{E(R+2(k+1)) \backslash E(R+2 k)} \exp (2 r) f^{2} \leq C 3^{-k}(R+2(k+1))
$$

which tends to 0 as $k \rightarrow \infty$. Hence

$$
\int_{E(R+2) \backslash E(R)} \exp (2 r) f^{2} \leq C .
$$

for some constant $C>0$ independent of $R$. The lemma then follows from (1.5).

q.e.d.

We point out that Lemma 1.1 also holds for any function $f$ with $a=0$ provided that $f$ is the limit of a sequence of harmonic functions $f_{R}$ on $E(R)$ satisfying $f_{R}=0$ on $\partial E(R)$ regardless of their boundary values on $\partial E$.

Lemma 1.2. Under the same assumption as in Lemma 1.1, the Dirichlet integral of the function $f$ must satisfy the decay estimate

$$
\int_{E(R+1) \backslash E(R)}|\nabla f|^{2} \leq C \exp \left(-2 \sqrt{\lambda_{1}(E)} R\right)
$$

and

$$
\int_{E(R)} \exp \left(2 \sqrt{\lambda_{1}(E)} r\right)|\nabla f|^{2} \leq C R
$$

for $R$ sufficiently large. 
Proof. For any $R>1$, let $\phi$ be a nonnegative cut-off function defined by

$$
\phi(x)= \begin{cases}r(x)-R+1 & \text { on } E(R) \backslash E(R-1) \\ 1 & \text { on } E(R+1) \backslash E(R) \\ R+2-r(x) & \text { on } E(R+2) \backslash E(R+1) .\end{cases}
$$

Integration by parts yields

$$
\begin{aligned}
0 & =\int_{E} \phi^{2}(f-a) \Delta(f-a) \\
& =-2 \int_{E} \phi(f-a)\langle\nabla \phi, \nabla f\rangle-\int_{E} \phi^{2}|\nabla f|^{2} .
\end{aligned}
$$

Together with the Schwarz inequality, this implies that

$$
\begin{aligned}
\int_{E} \phi^{2}|\nabla f|^{2} & =-2 \int_{E} \phi(f-a)\langle\nabla \phi, \nabla f\rangle \\
& \leq \frac{1}{2} \int_{E} \phi^{2}|\nabla f|^{2}+2 \int_{E}|\nabla \phi|^{2}(f-a)^{2},
\end{aligned}
$$

hence, by Lemma 1.1,

$$
\begin{aligned}
\int_{E(R+1) \backslash E(R)}|\nabla f|^{2} & \leq 4 \int_{E(R+2) \backslash E(R-1)}(f-a)^{2} \\
& \leq C \exp \left(-2 \sqrt{\lambda_{1}(E)} R\right),
\end{aligned}
$$

which is the first part of the lemma. The preceding inequality also implies that

$$
\int_{E(R+1) \backslash E(R)} \exp \left(2 \sqrt{\lambda_{1}(E)} r\right)|\nabla f|^{2} \leq C
$$

Setting $R=R_{0}+i$ and summing up over the inequalities for $1 \leq i \leq k$, we obtain

$$
\int_{E\left(R_{0}+k+1\right) \backslash E\left(R_{0}+1\right)} \exp \left(2 \sqrt{\lambda_{1}(E)} r\right)|\nabla f|^{2} \leq C k .
$$

This proves the desired estimate.

q.e.d.

Note that when applying Lemma 1.1 to the Green's function, we obtain the following sharp decay estimate. 
Corollary 1.3. Let $M$ be a complete manifold with $\lambda_{1}(M)>0$. Then the minimal positive Green's function $G(p, \cdot)$ with pole at $p \in M$ must satisfy the decay estimate

$$
\int_{B_{p}(R+1) \backslash B_{p}(R)} G^{2}(p, x) d x \leq C \exp \left(-2 \sqrt{\lambda_{1}(M)} R\right)
$$

for $R \geq 1$.

In the case when $M=\mathbb{H}^{n}$, the $n$-dimensional hyperbolic space with constant -1 sectional curvature, the Green's function is given by

$$
G(p, x)=C \int_{r(x)}^{\infty} \frac{d t}{A_{p}(t)}
$$

where $A_{p}(t)=\sinh ^{(n-1)} t$ is the area of the boundary of the geodesic ball of radius $t$ centered at $p \in \mathbb{H}^{n}$. One computes readily that the integral

$$
\int_{B_{p}(R+1) \backslash B_{p}(R)} G^{2}(p, x) d x \sim C \exp (-(n-1) R) .
$$

Since $\lambda_{1}\left(\mathbb{H}^{n}\right)=\frac{(n-1)^{2}}{4}$, the quantity $2 \sqrt{\lambda_{1}\left(\mathbb{H}^{n}\right)}$ is exactly $(n-1)$.

Applying Lemma 1.1, we will obtain volume estimates for those ends with positive spectrum. As pointed out in the introduction, these estimates are sharp. The sharp growth estimate is realized by the hyperbolic space $\mathbb{H}^{n}$, while the sharp decay estimate is realized by a hyperbolic cusp. Moreover, Example 2.2 in dimension 3 also realizes the sharp growth estimate on the non-parabolic end and the sharp decay estimate on the parabolic end. To state our estimate, let us denote the volume of the set $E(R)$ by $V_{E}(R)$. Analagously, the volume of the end $E$ will be denoted by $V_{E}(\infty)$.

Theorem 1.4. Let $E$ be an end of complete manifold $M$ with $\lambda_{1}(E)>0$.

(1) If $E$ is a parabolic end, then $E$ must have exponential volume decay given by

$$
V_{E}(\infty)-V_{E}(R) \leq C \exp \left(-2 \sqrt{\lambda_{1}(E)} R\right)
$$

for some constant $C>0$ depending on the end $E$. 
(2) If $E$ is a non-parabolic end, then $E$ must have exponential volume growth given by

$$
V_{E}(R) \geq C \exp \left(2 \sqrt{\lambda_{1}(E)} R\right)
$$

for all $R \geq R_{0}+1$ and some constant $C$ depending on the end $E$.

Proof. Again, we normalize $\lambda_{1}(E)=1$ by scaling the metric. Let $f_{R}$ be the harmonic function on $E(R)$ with $f=1$ on $\partial E$ and $f=0$ on $\partial E(R)$. The assumption that $E$ is parabolic implies that $f_{R}$ converges to $f=1$ as $R \rightarrow \infty$. Hence (1.5) becomes

$$
V_{E}(R+2)-V_{E}(R) \leq C \exp (-2 R)
$$

Letting $R=R+2 i$ for $i=0,1, \ldots$ and summing over $i$, we conclude that

$$
\begin{aligned}
V_{E}(\infty)-V_{E}(R) & \leq C \sum_{i=0}^{\infty} \exp (-2(R+2 i)) \\
& \leq C \exp (-2 R)
\end{aligned}
$$

This proves the volume decay estimate for the case of parabolic ends.

If $E$ is non-parabolic, then $f_{R}$ converges to a nonconstant harmonic function $f$ on $E$. Thus, we conclude that there exists a positive constant $C$ such that for $r \geq R_{0}$

$$
\begin{aligned}
C & =\int_{\partial E} \frac{\partial f}{\partial \nu} \\
& =\int_{\partial E(r)} \frac{\partial f}{\partial \nu} \\
& \leq \int_{\partial E(r)}|\nabla f| \\
& \leq A_{E}^{\frac{1}{2}}(r)\left(\int_{\partial E(r)}|\nabla f|^{2}\right)^{1 / 2},
\end{aligned}
$$

or equivalently,

$$
\frac{C}{A_{E}(r)} \leq \int_{\partial E(r)}|\nabla f|^{2}
$$


Integrating the preceding inequality with respect to $r$ from $R$ to $R+1$ and using Lemma 1.2, we obtain

$$
\begin{aligned}
\int_{R}^{R+1} \frac{1}{A_{E}(r)} d r & \leq C \int_{E(R+1) \backslash E(R)}|\nabla f|^{2} \\
& \leq C \exp (-2 R) .
\end{aligned}
$$

Therefore,

$$
\begin{aligned}
1 & \leq \int_{R}^{R+1} A_{E}(r) d r \int_{R}^{R+1} \frac{1}{A_{E}(r)} d r \\
& \leq C \exp (-2 R)\left(V_{E}(R+1)-V_{E}(R)\right) \\
& \leq C \exp (-2 R) V_{E}(R+1) .
\end{aligned}
$$

Since $R$ is arbitrary, we conclude that

$$
V_{E}(R) \geq C \exp (2 R)
$$

by adjusting the constant $C$. The theorem is proved.

q.e.d.

Our last corollary concerns the $L^{p}$ harmonic functions on ends with positive bottom spectrum.

Corollary 1.5. Let $E$ be an end of $M$ with $\lambda_{1}(E)>0$. Let $H^{p}(E)$ be the space of $L^{p}$ harmonic functions on $E$. When $p \geq 2$, if $u \in H^{p}(E)$, then $u$ must be bounded and it must satisfy the estimate

$$
\int_{E(R+1) \backslash E(R)} u^{2} \leq C \exp \left(-2 \sqrt{\lambda_{1}(E)} R\right) .
$$

When $1<p<2$, the same conclusion is true provided that the volume growth of $E$ is bounded by

$$
V_{E}(R) \leq C \exp \left(\frac{2 p}{2-p} \sqrt{\lambda_{1}(E)} R\right)
$$

Proof. Let $u \in H^{p}(E)$ be an $L^{p}$-harmonic function. Define $f_{R}$ to be the harmonic function on $E(R)$ satisfying

$$
f_{R}=0 \quad \text { on } \partial E(R)
$$

and

$$
f_{R}=u \quad \text { on } \partial E
$$


Clearly, the maximum principle asserts that a subsequence of $f_{R}$ as $R \rightarrow \infty$ will converge to a function $f \in H^{\infty}(E)$ with $u=f$ on $\partial E$. Moreover, by Lemma 1.1, $f$ satisfies the estimate

$$
\int_{E(R+1) \backslash E(R)} f^{2} \leq C \exp \left(-2 \sqrt{\lambda_{1}(E)} R\right) .
$$

If $p \geq 2$, the boundedness of $f$ implies that $f \in H^{p}(E)$. In particular, the function $u-f$ is in $H^{p}(E)$ with 0 boundary condition on $\partial E$. Applying the uniqueness theorem of Yau [19] for $L^{p}$ harmonic functions, we conclude that $u=f$.

For $1<p<2$, the Schwarz inequality, (1.6) and the volume growth bound give that

$$
\begin{aligned}
\int_{E(R+1) \backslash E(R)} f^{p} & \leq\left(\int_{E(R+1) \backslash E(R)} f^{2}\right)^{\frac{p}{2}}\left(V_{E}(R+1)-V_{E}(R)\right)^{\frac{2-p}{2}} \\
& \leq C \exp \left(-p \sqrt{\lambda_{1}(E)} R\right) \exp \left(p \sqrt{\lambda_{1}(E)} R\right) \\
& \leq C .
\end{aligned}
$$

This implies that the $L^{p}$-norm of $f$ is at most of linear growth. Again, by slightly modifying Yau's uniqueness theorem we conclude that $f=u$. Indeed for the completeness sake, we will outline a modification of Yau's argument for this case.

Observe that the function $g=|f-u|$ is a subharmonic function defined on $E$ with boundary condition

$$
g=0 \quad \text { on } \partial E \text {. }
$$

Let $\phi$ be a cut-off function satisfying

$$
\phi= \begin{cases}1 & \text { on } E(R) \\ 0 & \text { on } E \backslash E(2 R)\end{cases}
$$

and

$$
|\nabla \phi| \leq C R^{-1} \quad \text { on } E(2 R) \backslash E(R) .
$$

Integration by parts yields

$$
\begin{aligned}
0 & \leq \int_{E} \phi^{2} g^{p-1} \Delta g \\
& =-2 \int_{E} \phi g^{p-1}\langle\nabla \phi, \nabla g\rangle-(p-1) \int_{E} \phi^{2} g^{p-2}|\nabla g|^{2} .
\end{aligned}
$$


On the other hand, applying the Schwarz inequality

$$
-2 \int_{E} \phi g^{p-1}\langle\nabla \phi, \nabla g\rangle \leq \frac{p-1}{2} \int_{E} \phi^{2} g^{p-2}|\nabla g|^{2}+\frac{2}{p-1} \int_{E}|\nabla \phi|^{2} g^{p},
$$

we conclude that

$$
\int_{E} \phi^{2} g^{p-2}|\nabla g|^{2} \leq \frac{4}{(p-1)^{2}} \int_{E}|\nabla \phi|^{2} g^{p} .
$$

Using the property of $\phi$, this implies that

$$
\int_{E(R)} g^{p-2}|\nabla g|^{2} \leq \frac{C}{R^{2}} \int_{E(2 R) \backslash E(R)} g^{p} .
$$

The growth estimate on the $L^{p}$-norm of $f$ and the fact that $u \in L^{p}$ implies that the right hand side tends to 0 as $R \rightarrow \infty$. Hence $g$ must be identically constant. The boundary condition of $g$ asserts that it must be identically 0 , and $f=u$.

In both cases, since $f=u$, the function $u$ must satisfy (1.6). This concludes the corollary.

q.e.d.

\section{Splitting theorem}

We are now ready to prove the first main theorem.

Theorem 2.1. Let $M$ be a complete Riemannian manifold of dimension $n \geq 3$. Suppose $\lambda_{1}(M)>0$ and

$$
\operatorname{Ric}_{M} \geq-\frac{(n-1) \lambda_{1}(M)}{n-2}
$$

Then either

(1) $M$ has only one end with infinite volume; or

(2) $M=\mathbb{R} \times N$ with the warped product metric

$$
d s^{2}=d t^{2}+\cosh ^{2}\left(\sqrt{\frac{\lambda_{1}(M)}{n-2}} t\right) d s_{N}^{2},
$$

where $N$ is a compact manifold with Ricci curvature bounded from below by

$$
\operatorname{Ric}_{N} \geq-\lambda_{1}(M) .
$$


Proof. Let $f \in K$ be a harmonic function constructed in the previous section. Let us denote

$$
h=|\nabla f|
$$

to be the length of the gradient of $f$. The Bochner formula (see [9]) asserts that

$$
\Delta h^{2}=2 \operatorname{Ric}_{M}(\nabla f, \nabla f)+2\left|\nabla^{2} f\right|^{2},
$$

where $\operatorname{Ric}_{M}$ denotes the Ricci curvature of $M$ and $\nabla^{2} f$ is the Hessian of $f$. It was first observed by Yau [18] (also see [13]) that

$$
\left|\nabla^{2} f\right|^{2} \geq \frac{\left.n|\nabla| \nabla f\right|^{2}}{(n-1)|\nabla f|^{2}}
$$

For completeness sake, we will include an outline of this argument.

By choosing an orthonormal basis $\left\{e_{1}, e_{2}, \cdots, e_{n}\right\}$ such that $|\nabla f| e_{1}$ $=\nabla f$, and $e_{\alpha} f=0$ for all $\alpha \neq 1$, we have

$$
\begin{aligned}
\left|\nabla^{2} f\right| & =\sum_{i, j=1}^{n} f_{i j}^{2} \\
& \geq \sum_{j=1}^{n} f_{1 j}^{2}+\sum_{\alpha=2}^{n} f_{\alpha 1}^{2}+\sum_{\alpha=2}^{n} f_{\alpha \alpha}^{2} \\
& \geq \sum_{j=1}^{n} f_{1 j}^{2}+\sum_{\alpha=2}^{n} f_{\alpha 1}^{2}+\frac{1}{n-1}\left(\sum_{\alpha=2}^{n} f_{\alpha \alpha}\right)^{2} \\
& =\sum_{j=1}^{n} f_{1 j}^{2}+\sum_{\alpha=2}^{n} f_{\alpha 1}^{2}+\frac{1}{n-1} f_{11}^{2} \\
& \geq \frac{n}{n-1} \sum_{j=1}^{n} f_{1 j}^{2} .
\end{aligned}
$$

On the other hand,

$$
\begin{aligned}
\left.4|\nabla f|^{2}|\nabla| \nabla f\right|^{2} & =\left|\nabla\left(|\nabla f|^{2}\right)\right|^{2} \\
& =4 \sum_{j=1}^{n}\left(\sum_{i=1}^{n} f_{i} f_{i j}\right)^{2} \\
& =4 f_{1}^{2} \sum_{j=1}^{n} f_{1 j}^{2},
\end{aligned}
$$


hence

$$
\left|\nabla^{2} f\right| \geq\left.\frac{n}{n-1}|\nabla| \nabla f\right|^{2}
$$

Hence combining with the Bochner formula and the lower bound of the Ricci curvature, we have

$$
\Delta h \geq-\frac{(n-1) \lambda_{1}(M)}{(n-2)} h+\frac{|\nabla h|^{2}}{(n-1) h} .
$$

Setting $g=h^{\frac{n-2}{n-1}}=|\nabla f|^{\frac{n-2}{n-1}}$, this differential inequality can be rewritten as

$$
\Delta g \geq-\lambda_{1}(M) g .
$$

We now claim that the function $g$ must satisfy the integral condition

$$
\int_{B_{p}(2 R) \backslash B_{p}(R)} g^{2} \leq C R .
$$

To see this, let us apply the Schwarz inequality and get

$$
\begin{aligned}
\int_{B_{p}(2 R) \backslash B_{p}(R)} g^{2} & \left(\int_{B_{p}(2 R) \backslash B_{p}(R)} \exp \left(2 \sqrt{\lambda_{1}(M)} r\right)|\nabla f|^{2}\right)^{\frac{n-2}{n-1}} \\
\leq & \left(\int_{B_{p}(2 R) \backslash B_{p}(R)} \exp \left(-2(n-2) \sqrt{\lambda_{1}(M)} r\right)\right)^{\frac{1}{n-1}} .
\end{aligned}
$$

Using the lower bound of the Ricci curvature, the volume comparison theorem asserts that

$$
A_{p}(r) \leq A_{\kappa}(r)
$$

where $A_{\kappa}(r)$ is the area of the geodesic sphere of radius $r$ in the constant curvature space form with sectional curvature given by $\kappa=-\frac{\lambda_{1}(M)}{n-2}$. A direct computation yields that

$$
\begin{aligned}
& \int_{B_{p}(2 R) \backslash B_{p}(R)} \exp \left(-2(n-2) \sqrt{\lambda_{1}(M)} r\right) \\
& \leq C \int_{R}^{2 R} \exp \left(-2(n-2) \sqrt{\lambda_{1}(M)} r\right) \exp \left(\frac{(n-1) \sqrt{\lambda_{1}(M)}}{\sqrt{n-2}} r\right) d r \\
& =C \int_{R}^{2 R} \exp \left(\left(\frac{n-1}{\sqrt{n-2}}-2(n-2)\right) \sqrt{\lambda_{1}(M)} r\right) d r .
\end{aligned}
$$


The right hand side is at most linear in $R$ when $n=3$, and exponentially decays to 0 when $n \geq 4$. On the other hand, combining with the decay estimate in Lemma 1.2, the estimate (2.5) takes the form

$$
\int_{B_{p}(2 R) \backslash B_{p}(R)} g^{2} \leq C R
$$

for the case when $n=3$, and

$$
\int_{B_{p}(2 R) \backslash B_{p}(R)} g^{2} \rightarrow 0
$$

when $n \geq 4$. This proves our claim on the $L^{2}$ estimate of $g$.

To complete our proof of the theorem, we consider $\phi$ to be a nonnegative compactly supported function on $M$. Then

$$
\int_{M}|\nabla(\phi g)|^{2}=\int_{M}|\nabla \phi|^{2} g^{2}+2 \int_{M} \phi g\langle\nabla \phi, \nabla g\rangle+\int_{M} \phi^{2}|\nabla g|^{2} .
$$

The second term on the right hand side can be written as

$$
\begin{aligned}
& 2 \int_{M} \phi g\langle\nabla \phi, \nabla g\rangle \\
& =\frac{1}{2} \int_{M}\left\langle\nabla\left(\phi^{2}\right), \nabla\left(g^{2}\right)\right\rangle \\
& =-\int_{M} \phi^{2} g \Delta g-\int_{M} \phi^{2}|\nabla g|^{2} \\
& =\lambda_{1}(M) \int_{M} \phi^{2} g^{2}-\int_{M} \phi^{2}|\nabla g|^{2}-\int_{M} \phi^{2} g\left(\Delta g+\lambda_{1}(M) g\right) .
\end{aligned}
$$

Combining with (2.6) and the variational principle of $\lambda_{1}(M)$, this implies that

$$
\begin{aligned}
\lambda_{1}(M) \int_{M} \phi^{2} g^{2} \leq & \int_{M}|\nabla(\phi g)|^{2} \\
= & \lambda_{1}(M) \int_{M} \phi^{2} g^{2}+\int_{M}|\nabla \phi|^{2} g^{2} \\
& -\int_{M} \phi^{2} g\left(\Delta g+\lambda_{1}(M) g\right) .
\end{aligned}
$$

Hence, we have

$$
\int_{M} \phi^{2} g\left(\Delta g+\lambda_{1}(M) g\right) \leq \int_{M}|\nabla \phi|^{2} g^{2} .
$$


For $R>0$, let us choose $\phi$ to satisfy the properties that

$$
\phi= \begin{cases}1 & \text { on } B_{p}(R) \\ 0 & \text { on } M \backslash B_{p}(2 R)\end{cases}
$$

and

$$
|\nabla \phi| \leq C R^{-1} \quad \text { on } B_{p}(2 R) \backslash B_{p}(R)
$$

for some constant $C>0$. Then the right hand side of (2.8) can be estimated by

$$
\int_{M}|\nabla \phi|^{2} g^{2} \leq C R^{-2} \int_{B_{p}(2 R) \backslash B_{p}(R)} g^{2} .
$$

By the $L^{2}$ estimate of $g$, this tends to 0 as $R \rightarrow \infty$. Hence, by (2.4), we conclude that $g$ either must be identically 0 or it must satisfy

$$
\Delta g=-\lambda_{1}(M) g
$$

If $M$ has more than one infinite volume end, then by the discussion in $\S 1$ there must exist a nonconstant $f$, hence $g \neq 0$. So all the inequalities used in deriving (2.2) become equality. We can now recall the argument in [15] (also [16]) to conclude that $M=\mathbb{R} \times N$ with the warped product metric $d s^{2}=d t^{2}+\cosh ^{2}\left(\sqrt{\frac{\lambda_{1}(M)}{n-2}} t\right) d s_{N}^{2}$ for some compact manifold $N$ with $\operatorname{Ric}_{N} \geq-\lambda_{1}(M)$.

Indeed, since $\Delta f=0$, the Hessian of $f$ must be of the form

$$
\left(f_{i j}\right)=\left(\begin{array}{ccccc}
-(n-1) \mu & 0 & 0 & \ldots & 0 \\
0 & \mu & 0 & \ldots & 0 \\
0 & 0 & \mu & \ldots & 0 \\
& & & \ddots & \\
0 & 0 & 0 & \ldots & \mu
\end{array}\right) .
$$

The fact that $f_{1 \alpha}=0$ for all $\alpha \neq 1$ implies that $|\nabla f|$ is identically constant along the level set of $f$. In particular, the level sets of $|\nabla f|$ and $f$ coincide. Moreover,

$$
\begin{aligned}
\mu \delta_{\alpha \beta} & =f_{\alpha \beta} \\
& =h_{\alpha \beta} f_{1}
\end{aligned}
$$


with $\left(h_{\alpha \beta}\right)$ being the second fundamental form of the level set of $f$. Hence

$$
f_{11}=-H f_{1}
$$

where $H$ is the mean curvature of the level set of $f$. Applying the same computation on the function $g$, we obtain

$$
\begin{aligned}
-\lambda_{1}(M) g & =\Delta g \\
& =g_{11}+H g_{1} .
\end{aligned}
$$

On the other hand, since $g=|\nabla f|^{\frac{n-2}{n-1}}$, we have

$$
\begin{aligned}
g_{1} & =\left(|\nabla f|^{\frac{n-2}{n-1}}\right)_{1} \\
& =\frac{n-2}{n-1}|\nabla f|^{-\frac{n}{n-1}} f_{i} f_{i 1} \\
& =\frac{n-2}{n-1} f_{1}^{-\frac{1}{n-1}} f_{11} .
\end{aligned}
$$

Hence, combining with (2.9), we conclude that

$$
\begin{aligned}
H & =-f_{1}^{-1} f_{11} \\
& =-\frac{n-1}{n-2} g_{1} g^{-1} .
\end{aligned}
$$

Substituting into (2.10), this yields

$$
g_{11}-\frac{n-1}{n-2}\left(g_{1}\right)^{2} g^{-1}+\lambda_{1}(M) g=0 .
$$

Setting $u=g^{-\frac{1}{n-2}}=|\nabla f|^{-\frac{1}{n-1}}$, this differential equation becomes

$$
u_{11}-\frac{\lambda_{1}(M)}{n-2} u=0 .
$$

Viewing this as an ODE along the integral curve generated by the vector field $e_{1}$, one concludes that

$$
u(t)=A \exp \left(\sqrt{\frac{\lambda_{1}(M)}{n-2}} t\right)+B \exp \left(-\sqrt{\frac{\lambda_{1}(M)}{n-2}} t\right) .
$$

Since $u$ must be nonnegative, $A$ and $B$ must be nonnegative. Moreover, $\nabla f \neq 0$. 
Note that $M$ is assumed to have at least two infinite volume ends. We claim that any fixed level set $N$ of $h$ must be compact. Indeed, by the fact that $f$ has no critical points and that the level set of $f$ coincides with the level set of $h, M$ must be topologically the product $\mathbb{R} \times N$. If $N$ is noncompact then $M$ will have only one end, hence $N$ must be compact. Since $h=|\nabla f|$ is in $L^{2}$, we conclude that $h$ must have an interior maximum, say $h=1$. We now fix $N=\{h=1\}$. Then the function $u$ must have its minimum along $N$, hence by reparameterizing, we may assume $N$ is given by $t=0$. Therefore,

$$
0=u^{\prime}(0)=A-B \text {. }
$$

and

$$
1=u(0)=A+B
$$

This implies that

$$
u(t)=\cosh \left(\sqrt{\frac{\lambda_{1}(M)}{n-2}} t\right)
$$

and

$$
g(t)=\cosh ^{-(n-2)}\left(\sqrt{\frac{\lambda_{1}(M)}{n-2}} t\right) .
$$

Using (2.11), we conclude that

$$
H(t)=(n-1) \tanh \left(\sqrt{\frac{\lambda_{1}(M)}{n-2}} t\right)
$$

and

$$
\left(h_{\alpha \beta}(t)\right)=\tanh \left(\sqrt{\frac{\lambda_{1}(M)}{n-2}} t\right) .
$$

This implies that the metric on $M=\mathbb{R} \times N$ must be of the form

$$
d s_{M}=d t^{2}+\cosh ^{2}\left(\sqrt{\frac{\lambda_{1}(M)}{n-2}} t\right) d s_{N}^{2}
$$

as claimed.

q.e.d.

\section{Example 2.2.}

Let us consider the $n$ dimensional manifold $M=\mathbb{R} \times N$ endowed with the warped product metric

$$
d s_{M}^{2}=d t^{2}+\exp (2 t) d s_{N}^{2}
$$


where $d s_{N}^{2}$ is a metric on $N$ with nonnegative Ricci curvature. One computes directly that $M$ has Ricci curvature satisfying

$$
\operatorname{Ric}_{M} \geq-(n-1) .
$$

Moreover, the function $f=\exp (-(n-1) t)$ is a harmonic function on $M$. The length of its gradient is given by $h=(n-1) \exp (-(n-1) t)$. In particular, the function $g=h^{\frac{n-2}{n-1}}$ satisfies the equation

$$
\Delta g=-(n-2) g .
$$

Since $g$ is positive, this implies that $\lambda_{1}(M) \geq n-2$. This example indicates that it is possible that the class of manifolds considered in Theorem 2.1 has finite volume ends that are not being detected by our method.

\section{Finiteness theorem}

Theorem 3.1. Let $M$ be a complete Riemannian manifold of dimension $n \geq 3$. Suppose that there exists a geodesic ball $B_{p}\left(R_{0}\right) \subset M$ centered at $p \in M$ of radius $R_{0}$ such that the lower bound of the spectrum for the Dirichlet Laplacian on $M \backslash B_{p}\left(R_{0}\right)$ satisfies $\lambda_{1}\left(M \backslash B_{p}\left(R_{0}\right)\right)>0$. If the Ricci curvature is bounded from below by

$$
\operatorname{Ric}_{M} \geq-\frac{(n-1) \lambda_{1}\left(M \backslash B_{p}\left(R_{0}\right)\right)}{n-2}+\epsilon
$$

for some $\epsilon>0$ on $M \backslash B_{p}\left(R_{0}\right)$, then $M$ must have finitely many ends with infinite volume. In particular, there exists a constant $C\left(n, R_{0}, \alpha, v, \epsilon\right)>$ 0 depending on the quantities $n, R_{0}, \epsilon, \alpha=\inf _{B_{p}\left(3 R_{0}\right)} \operatorname{Ric}_{M}$, and $v=$ $\inf _{x \in B_{p}\left(2 R_{0}\right)} V_{x}\left(R_{0}\right)$, such that the number of infinite volume ends of $M$ is at most $C$.

Proof. In view of the discussion in $\S 1$, it suffices to estimate the dimension of the space $K$. Since we assume that $\lambda_{1}\left(M \backslash B_{p}\left(R_{0}\right)\right)>0$, Lemma 1.1 and Lemma 1.2 imply that for each $f \in K$, the function

$$
g=|\nabla f|^{\frac{n-2}{n-1}}
$$

must satisfy the estimate

$$
\int_{B_{p}(2 R) \backslash B_{p}(R)} g^{2} \leq C R .
$$


Following the proof of Theorem 2.1, the function $g$ satisfies the differential inequality

$$
\Delta g \geq\left(\epsilon-\lambda_{1}\left(M \backslash B_{p}\left(R_{0}\right)\right)\right) g
$$

on $M \backslash B_{p}\left(R_{0}\right)$. Moreover (2.8) in the form

$$
\epsilon \int_{M} \phi^{2} g^{2} \leq \int_{M}|\nabla \phi|^{2} g^{2}
$$

is valid for any nonnegative cut-off function $\phi$ with support in $M \backslash$ $B_{p}\left(R_{0}\right)$. Let us choose $\phi$ to satisfy

$$
\begin{gathered}
\phi= \begin{cases}0 & \text { on } B_{p}\left(R_{0}\right) \\
1 & \text { on } B_{p}(R) \backslash B_{p}\left(2 R_{0}\right) \\
0 & \text { on } M \backslash B_{p}(2 R),\end{cases} \\
|\nabla \phi| \leq C R_{0}^{-1} \quad \text { on } B_{p}\left(2 R_{0}\right) \backslash B_{p}\left(R_{0}\right),
\end{gathered}
$$

and

$$
|\nabla \phi| \leq C R^{-1} \quad \text { on } B_{p}(2 R) \backslash B_{p}(R)
$$

for some constant $C>0$. Arguing as in the proof of Theorem 2.1, using the fact that

$$
\int_{B_{p}(2 R) \backslash B_{p}(R)} g^{2} \leq C R,
$$

and letting $R \rightarrow \infty$, we conclude from (3.2) that

$$
\epsilon \int_{M \backslash B_{p}\left(2 R_{0}\right)} g^{2} \leq C R_{0}^{-2} \int_{B_{p}\left(2 R_{0}\right) \backslash B_{p}\left(R_{0}\right)} g^{2} .
$$

In particular,

$$
\int_{B_{p}\left(3 R_{0}\right)} g^{2} \leq\left(1+\frac{C}{\epsilon R_{0}^{2}}\right) \int_{B_{p}\left(2 R_{0}\right)} g^{2} .
$$

Since the function $g$ satisfies the differential inequality

$$
\Delta g \geq-\alpha g
$$

on $B_{p}\left(3 R_{0}\right)$, the mean value inequality of Li-Tam [11] asserts that

$$
\begin{aligned}
g^{2}(x) & \leq C \int_{B_{x}\left(R_{0}\right)} g^{2} \\
& \leq C \int_{B_{p}\left(3 R_{0}\right)} g^{2}
\end{aligned}
$$


for any $x \in B_{p}\left(2 R_{0}\right)$, where $C>0$ is a constant depending only on $n$, $\alpha$, and $v$. Combining with (3.3), this implies that

$$
\sup _{B_{p}\left(2 R_{0}\right)} g^{2} \leq C \int_{B_{p}\left(2 R_{0}\right)} g^{2}
$$

On the other hand, the Schwarz inequality asserts that

$$
\int_{B_{p}\left(2 R_{0}\right)} g^{2} \leq\left(\int_{B_{p}\left(2 R_{0}\right)}|\nabla f|^{2}\right)^{\frac{n-2}{n-1}} V_{p}\left(2 R_{0}\right)^{\frac{1}{n-1}}
$$

Hence,

$$
\sup _{B_{p}\left(2 R_{0}\right)}|\nabla f|^{2} \leq C \int_{B_{p}\left(2 R_{0}\right)}|\nabla f|^{2} .
$$

Note that if $f$ is not identically constant then unique continuation implies that

$$
\int_{B_{p}\left(2 R_{0}\right)}|\nabla f|^{2} \neq 0
$$

Hence, the bilinear form

$$
\int_{B_{p}\left(2 R_{0}\right)}\left\langle\nabla f_{1}, \nabla f_{2}\right\rangle
$$

is nondegenerate on the space of 1 -forms

$$
\bar{K}=\{d f \mid f \in K\} .
$$

Applying Lemma 11 of [7], there exists $d f_{0} \in \bar{K} \backslash\{0\}$ such that

$$
\operatorname{dim} \bar{K} \int_{B_{p}\left(2 R_{0}\right)}\left|d f_{0}\right|^{2} \leq V_{p}\left(2 R_{0}\right)(\min \{n, \operatorname{dim} \bar{K}\}) \sup _{B_{p}\left(2 R_{0}\right)}\left|d f_{0}\right|^{2} .
$$

However, combining with (3.4) we conclude that

$$
\operatorname{dim} K=\operatorname{dim} \bar{K}+1 \leq C .
$$




\section{Estimating the dimension of $H^{1}\left(L^{2}(M)\right)$}

If we assume a more restrictive hypothesis on the Ricci curvature, it is possible to estimate the dimension of the space of $L^{2}$ harmonic 1forms, $H^{1}\left(L^{2}(M)\right)$. As pointed out in [13], since the exterior differential of a harmonic function with finite Dirichlet integral is an $L^{2}$ harmonic 1-form, we have

$$
\operatorname{dim} H^{1}\left(L^{2}(M)\right)+1 \geq \text { number of non-parabolic ends. }
$$

In addition, if $\lambda_{1}(M)>0$, then

$$
\operatorname{dim} H^{1}\left(L^{2}(M)\right)+1 \geq \text { number of infinite volume ends. }
$$

Hence, in general, an estimate on $\operatorname{dim} H^{1}\left(L^{2}(M)\right)$ is stronger than an estimate on the number of ends with infinite volume. Let us first establish the following lemma which may be useful in other similar situations.

Lemma 4.1. Let $M$ be a complete Riemannian manifold. Suppose $h$ is a nonnegative function satisfying the differential inequality

$$
\Delta h \geq-a h+b \frac{|\nabla h|^{2}}{h}
$$

where $a$ and $b$ are constants with $b \geq 0$. Then for any $\delta>0$ and any compactly supported cut-off function $\phi$, we have

$$
\begin{aligned}
\int_{M}|\nabla(\phi h)|^{2} \leq & \frac{a(1+\delta)}{1+\delta(1+b)} \int_{M} \phi^{2} h^{2} \\
& +\left(1+\frac{\delta^{2} b}{1+\delta(1+b)}\right) \int_{M}|\nabla \phi|^{2} h^{2} .
\end{aligned}
$$

Proof. Let us consider the integral

$$
\int_{M}|\nabla(\phi h)|^{2}=\int_{M}|\nabla \phi|^{2} h^{2}+2 \int_{M} \phi h\langle\nabla \phi, \nabla h\rangle+\int_{M} \phi^{2}|\nabla h|^{2}
$$

The second term on the right hand side can be estimated by

$$
\begin{aligned}
2 \int_{M} \phi h\langle\nabla \phi, \nabla h\rangle & =-\int_{M} \phi^{2} h \Delta h-\int_{M} \phi^{2}|\nabla h|^{2} \\
& \leq a \int_{M} \phi^{2} h^{2}-(1+b) \int_{M} \phi^{2}|\nabla h|^{2} .
\end{aligned}
$$


On the other hand, it also can be estimated by

$$
2 \int_{M} \phi h\langle\nabla \phi, \nabla h\rangle \leq \delta \int_{M}|\nabla \phi|^{2} h^{2}+\frac{1}{\delta} \int_{M} \phi^{2}|\nabla h|^{2} .
$$

Combining the two estimates (4.2) and (4.3), we have

$$
\begin{aligned}
2 \int_{M} \phi h\langle\nabla \phi, \nabla h\rangle= & \left(\frac{2(1+\delta)}{1+\delta(1+b)}+\frac{2 \delta b}{1+\delta(1+b)}\right) \int_{M} \phi h\langle\nabla \phi, \nabla h\rangle \\
\leq & \frac{a(1+\delta)}{1+\delta(1+b)} \int_{M} \phi^{2} h^{2}+\frac{\delta^{2} b}{1+\delta(1+b)} \int_{M}|\nabla \phi|^{2} h^{2} \\
& -\int_{M} \phi^{2}|\nabla h|^{2} .
\end{aligned}
$$

The lemma follows by substituting this into (4.1).

q.e.d.

Theorem 4.2. Let $M$ be a complete Riemannian manifold. Suppose $\lambda_{1}(M)>0$ and

$$
\operatorname{Ric}_{M} \geq-\frac{n \lambda_{1}(M)}{n-1}+\epsilon
$$

for some $\epsilon>0$. Then $H^{1}\left(L^{2}(M)\right)=0$.

Proof. Let $\omega \in H^{1}\left(L^{2}(M)\right)$ be an $L^{2}$ harmonic 1-form. It is known that $\omega$ must be both closed and co-closed. In particular, the length of $\omega, h=|\omega|$, must satisfy the Bochner formula

$$
\Delta h \geq \frac{\operatorname{Ric}_{M}(\omega, \omega)}{h}+\frac{|\nabla h|^{2}}{(n-1) h} .
$$

Using the lower bound of the Ricci curvature, we conclude that

$$
\Delta h \geq\left(\epsilon-\frac{n \lambda_{1}(M)}{n-1}\right) h+\frac{|\nabla h|^{2}}{(n-1) h} .
$$

Let $\phi$ be a nonnegative cut-off function with compact support in $M$. By letting $a=\frac{n \lambda_{1}(M)}{n-1}-\epsilon$ and $b=\frac{1}{n-1}$, Lemma 4.1 asserts that

$$
\begin{aligned}
\int_{M}|\nabla(\phi h)|^{2} \leq & \left(\frac{n(1+\delta) \lambda_{1}(M)}{(n-1)+\delta n}-\frac{\epsilon(n-1)(1+\delta)}{(n-1)+\delta n}\right) \int_{M} \phi^{2} h^{2} \\
& +\frac{(n-1)+\delta(\delta+n)}{(n-1)+\delta n} \int_{M}|\nabla \phi|^{2} h^{2} .
\end{aligned}
$$


However, the variational principle of $\lambda_{1}(M)$ asserts that

$$
\lambda_{1}(M) \int_{M} \phi^{2} h^{2} \leq \int_{M}|\nabla(\phi h)|^{2} .
$$

Combining with (4.4), we conclude that

$$
\left(\epsilon(n-1)(1+\delta)-\lambda_{1}(M)\right) \int_{M} \phi^{2} h^{2} \leq((n-1)+\delta(\delta+n)) \int_{M}|\nabla \phi|^{2} h^{2} .
$$

For $R>0$, let us choose $\phi$ to satisfy the properties that

$$
\phi= \begin{cases}1 & \text { on } B_{p}(R) \\ 0 & \text { on } M \backslash B_{p}(2 R)\end{cases}
$$

and

$$
|\nabla \phi| \leq C R^{-1} \quad \text { on } B_{p}(2 R) \backslash B_{p}(R)
$$

for some constant $C>0$. Hence, (4.5) becomes

$$
\begin{aligned}
(\epsilon(n-1)(1+\delta) & \left.-\lambda_{1}(M)\right) \int_{B_{p}(R)} h^{2} \\
& \leq C R^{-2}((n-1)+\delta(\delta+n)) \int_{B_{p}(2 R) \backslash B_{p}(R)} h^{2} .
\end{aligned}
$$

Letting $R \rightarrow \infty$, the right hand side tends to 0 since $h \in L^{2}$. This implies that $h$ must be identically 0 by choosing $\delta$ sufficiently large so that $\epsilon(n-1)(1+\delta)>\lambda_{1}(M)$. This proves the vanishing of $H^{1}\left(L^{2}(M)\right)$.

q.e.d.

By combining the argument in the proof of Theorem 3.1 and Theorem 4.2 , we obtain the following theorem by assuming the lower bound of the Ricci curvature outside a compact set.

Theorem 4.3. Let $M$ be a complete Riemannian manifold. Suppose there exists a geodesic ball $B_{p}\left(R_{0}\right) \subset M$ such that $\lambda_{1}\left(M \backslash B_{p}\left(R_{0}\right)\right)>$ 0 , and

$$
\operatorname{Ric}_{M} \geq-\frac{n \lambda_{1}\left(M \backslash B_{p}\left(R_{0}\right)\right)}{n-1}+\epsilon
$$

on $M \backslash B_{p}\left(R_{0}\right)$ for some $\epsilon>0$. Then $H^{1}\left(L^{2}(M)\right)$ must be of finite dimension. In particular, there exists a constant $C\left(n, R_{0}, \alpha, v, \epsilon\right)>0$ depending on the quantities $n, R_{0}, \epsilon, \alpha=\inf _{B_{p}\left(3 R_{0}\right)} \operatorname{Ric}_{M}$, and $v=$ $\inf _{x \in B_{p}\left(2 R_{0}\right)} V_{x}\left(R_{0}\right)$, such that

$$
\operatorname{dim} H^{1}\left(L^{2}(M)\right) \leq C .
$$




\section{Kähler manifolds}

When the manifold $M$ is Kähler, one can prove that the manifold must only have one infinite volume end with a weaker curvature assumption than stated in Theorem 2.1. In this situation, the warped product example does not exist since it is not Kähler.

Theorem 5.1. Let $M$ be a complete Kähler manifold of complex dimension $m$. Let $x_{0}$ be the unique positive solution to the cubic

$$
4 x^{3}+2(2 m-1) x^{2}-(2 m-1)^{2}=0 .
$$

Suppose $\lambda_{1}(M)>0$ and

$$
\operatorname{Ric}_{M} \geq-(2 m-1) x_{0}^{-2} \lambda_{1}(M)+\epsilon
$$

for some $\epsilon>0$. Then $M$ must have only one end with infinite volume.

Proof. For convenience sake, let us first normalize the metric such that

$$
\inf _{M} \operatorname{Ric}_{M}=-(n-1),
$$

where $n=2 m$ is the real dimension of $M$. Following the proof of Theorem 2.1, we consider a harmonic function $f \in K$ and its gradient $h=|\nabla f|$. Since $f$ has finite Dirichlet integral, it was proved in Lemma 3.1 of [8] that it must be pluriharmonic. On the other hand, the Bochner formula for pluriharmonic function (see [8]) becomes

$$
\Delta h \geq-(n-1) h+\frac{|\nabla h|^{2}}{h} .
$$

If we let $g=h^{p}, 0<p<1$, then by an argument similar to (2.5), and the volume comparison theorem, we have

$$
\begin{aligned}
& \int_{B_{p}(2 R) \backslash B_{p}(R)} g^{2} \\
& \quad \leq C R^{p}\left(\int_{R}^{2 R} \exp \left(-\frac{p}{1-p} 2 \sqrt{\lambda_{1}(M) r}\right) \exp ((n-1) r) d r\right)^{1-p} .
\end{aligned}
$$

If we choose $p$ such that

$$
2 p \sqrt{\lambda_{1}(M)}=(1-p)(n-1),
$$

then

$$
\int_{B_{p}(R)} g^{2}=O(R)
$$


Moreover, since (5.1) implies that $g=h^{p}$ satisfies

$$
\Delta g \geq-p(n-1) g+\frac{|\nabla g|^{2}}{g}
$$

by Lemma 4.1, we obtain

$$
\left(\lambda_{1}(M)-\frac{p(n-1)(1+\delta)}{1+2 \delta}\right) \int_{M} \phi^{2} g^{2} \leq\left(1+\frac{\delta^{2}}{1+2 \delta}\right) \int_{M}|\nabla \phi|^{2} g^{2}
$$

for all $\delta>0$. Now if we have

$$
\lambda_{1}(M)>\frac{p(n-1)}{2},
$$

then there exists a sufficiently large $\delta$ such that

$$
\lambda_{1}(M)-\frac{p(n-1)(1+\delta)}{1+2 \delta}>0 .
$$

Arguing as in Theorem 4.2, we conclude that $g=0$ and $M$ has only one infinite volume end. However, condition (5.2) for $p$ asserts that

$$
p=\frac{n-1}{n-1+2 \sqrt{\lambda_{1}(M)}}
$$

hence we need

$$
2 \lambda_{1}(M)\left(n-1+2 \sqrt{\lambda_{1}(M)}\right)-(n-1)^{2}>0 .
$$

On the other hand, since the function

$$
q(x)=4 x^{3}+2(n-1) x^{2}-(n-1)^{2}
$$

is strictly increasing when $x>0$ with $q(0)<0$, (5.3) will be fulfilled as long as $\lambda_{1}(M)>x_{0}^{2}$, where $x_{0}>0$ is the positive solution to the cubic

$$
4 x^{3}+2(n-1) x^{2}-(n-1)^{2}=0 .
$$

This proves the theorem.

q.e.d.

We would like to point out that because of the Cheng's estimate asserting that

$$
\lambda_{1}(M) \leq \frac{(2 m-1)^{2}}{4}
$$


when $\inf _{M} \operatorname{Ric}_{M}=-(2 m-1)$, the hypothesis of Theorem 5.1 is ineffective when $m=1$. However, when $m$ is large, $x_{0}^{2}$ is much smaller than

$\frac{2 m-1}{2}$. In fact, because $q\left(\frac{\sqrt{n-1}}{\sqrt{2}}\right)>0$, we deduce that $x_{0}^{2}<\frac{n-1}{2}$. This is already better than the bound $n-2=2 m-2$ used in the hypothesis of Theorem 2.1 when $m \geq 2$. Obviously, a theorem similar to Theorem 3.1 is also valid for the Kähler case.

Theorem 5.2. Let $M$ be a complete Kähler manifold of complex dimension $m$. Let $x_{0}$ be the unique positive solution to the cubic

$$
4 x^{3}+2(2 m-1) x^{2}-(2 m-1)^{2}=0 .
$$

Suppose there exists a geodesic ball $B_{p}\left(R_{0}\right) \subset M$ such that $\lambda_{1}(M \backslash$ $\left.B_{p}\left(R_{0}\right)\right)>0$ and

$$
\operatorname{Ric}_{M} \geq-(2 m-1) x_{0}^{-2} \lambda_{1}\left(M \backslash B_{p}\left(R_{0}\right)\right)+\epsilon
$$

on $M \backslash B_{p}\left(R_{0}\right)$ for some $\epsilon>0$. Then $M$ must have finitely many ends with infinite volume. In particular, there exists a constant $C\left(m, R_{0}, \alpha, v, \epsilon\right)>0$ depending on the quantities $n, R_{0}, \epsilon, \alpha=$ $\inf _{B_{p}\left(3 R_{0}\right)} \operatorname{Ric}_{M}$, and $v=\inf _{x \in B_{p}\left(2 R_{0}\right)} V_{x}\left(R_{0}\right)$, such that the number of infinite volume ends of $M$ is at most $C$.

\section{References}

[1] J. Cheeger \& D. Gromoll, The splitting theorem for manifolds of nonnegative Ricci curvature, J. Differential Geom. 6 (1971) 119-128.

[2] S.Y. Cheng, Eigenvalue Comparison theorems and its Geometric Application, Math. Z. 143 (1975) 289-297.

[3] M. Cai \& G.J. Galloway, Boundaries of zero scalar curvature in the ADS/CFT correspondence, preprint hep-th/0003046.

[4] H. Cao, Y. Shen, \& S. Zhu, The structure of stable minimal hypersurfaces in $\mathbb{R}^{n+1}$, Math. Res. Let. 4 (1997) 637-644.

[5] J. Lee, The spectrum of an asymptotic hyperbolic Einstein Manifold, Comm. Anal. Geom. 3 (1995) 253-271.

[6] N.C. Leung \& T. Wan, Harmonic maps and the topology of conformally compact Einstein manifolds, Math. Res. Let., to appear.

[7] P. Li, On the Sobolev constant and the p-spectrum of a compact Riemannian manifold, Ann. Scient. Éc. Norm. Sup. 4, T 13 (1980) 451-469. 
[8] P. Li, On the structure of complete Kähler manifolds with nonnegative curvature near infinity, Invent. Math. 99 (1990) 579-600.

[9] P. Li, Lecture Notes on Geometric Analysis, in 'Lecture Notes Series 6 - Research Institute of Mathematics and Global Analysis Research Center,' Seoul National University, Seoul, 1993.

[10] P. Li, Curvature and function theory on Riemannian manifolds, in 'Survey in Differential Geometry “In Honor of Atiyah, Bott, Hirzebruch, and Singer", ' International Press, Cambridge VII (2000) 71-111.

[11] P. Li \& L.F. Tam, The heat equation and harmonic maps of complete manifolds, Invent. Math. 105 (1991) 1-46.

[12] P. Li \& L.F. Tam, Harmonic functions and the structure of complete manifolds, J. Differential Geom. 35 (1992) 359-383.

[13] P. Li \& J. Wang, Minimal hypersurfaces with finite index, Math. Res. Let., to appear.

[14] R. Mazzeo, The Hodge cohomology of a conformally compact metric, J. Differential Geom. 28 (1988) 309-339.

[15] X. Wang, On the geometry of conformally compact Einstein manifolds, Stanford Thesis, 2001.

[16] X. Wang, On conformally compact Einstein manifolds, Math. Res. Let., to appear.

[17] E. Witten \& S.T. Yau, Connectedness of the boundary in the AdS/CFT correspondence, Adv. Theor. Math. Phys. 3 (1999) 1635-1655.

[18] S.T. Yau, Harmonic functions on complete Riemannian manifolds, Comm. Pure Appl. Math. 28 (1975) 201-228.

[19] S.T. Yau, Some function-theoretic properties of complete Riemannian manifolds and their applications to geometry, Indiana Math. J. 25 (1976) 659-670.

UNIVERSITY OF CALIFORNIA

IRVINE, CA 92697-3875

UNIVERSITY OF MINNESOTA

MinNEAPOLIS, MN 55455 